$\mathrm{S}$, dans laquelle une pompe $\mathrm{P}$ déverse continuellement, par $\mathrm{E}$, de l'acide refroidi en $\mathrm{K}$ et puisé dans le récipient $\mathrm{V}$. De celui-ci, les oxydes de l'azore sont distillés par chauffage de l'acide et condensés en $\mathrm{C}$, d'où ils sortent en $\mathrm{F}$ où on les recueille.

Le montage des nombreux petits transformateurs nécessaires est coùteux et mal commode. L'inventeur indique pour cela une seconde disposition qui n'offre rien de nouveau au point de vue électrique et nous paraît, si belle soit-elle estimée, moins propre que la première à assurer une marche sans à-coup. Nous renonçons à la reproduire.

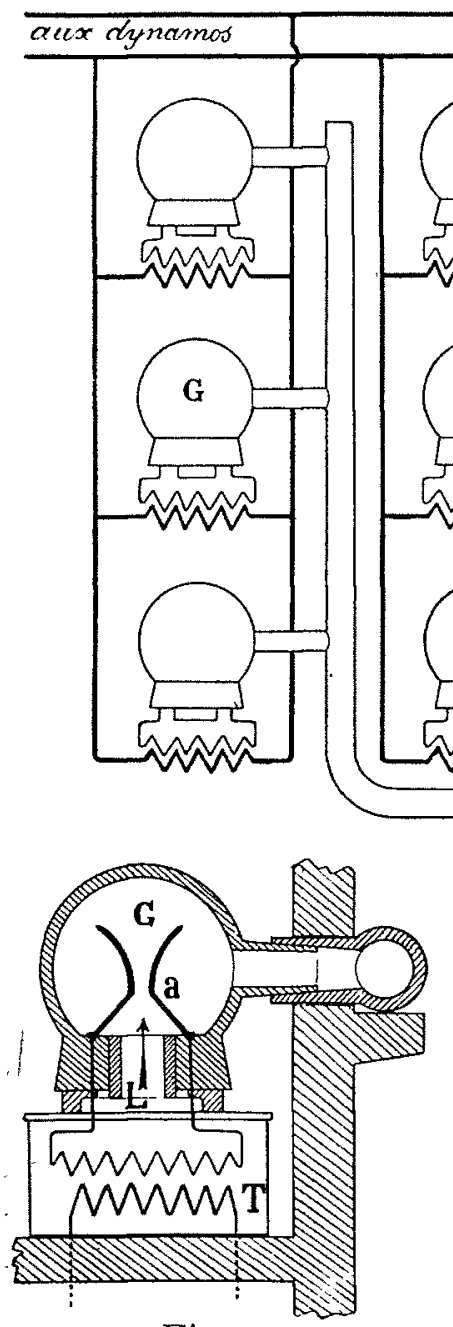

Fig. I

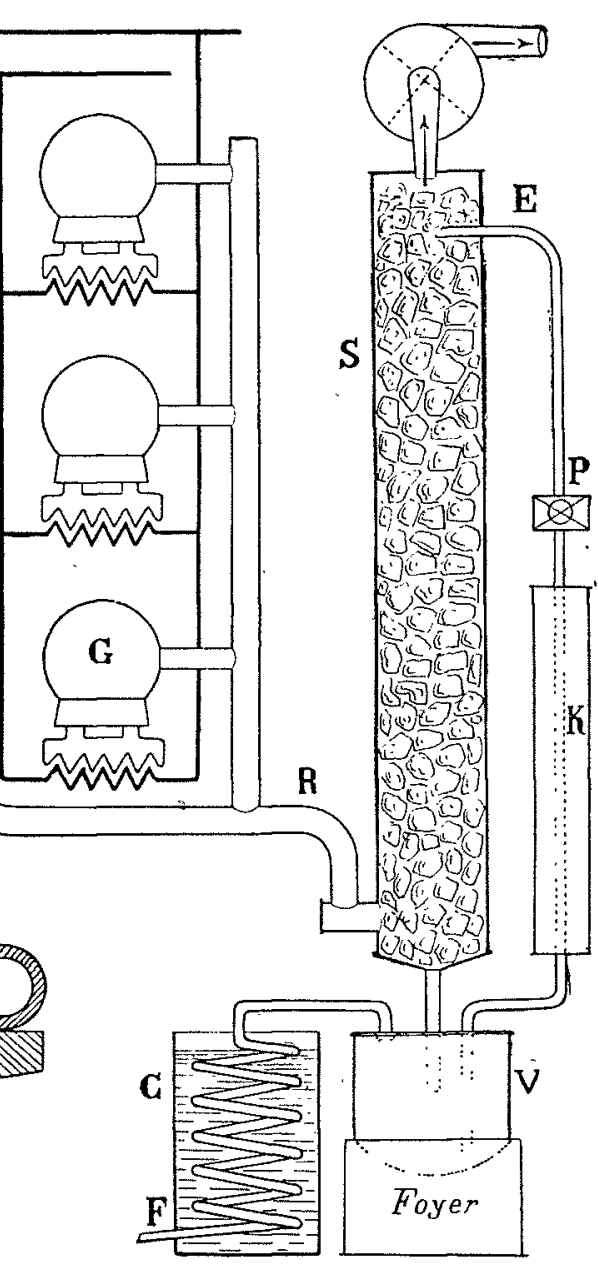

Fig. 2
La vitesse de l'air doit être réglée de manière à ne contenir que $3 \%$ de gaz nitreux. Les rendements sont alors les plus favorables.

Wilbert propose'les gaz des hauts fourneaux comme source d'énergie pour cette réaction.

Pour les raisons indiquées plus haut, les recherchés de Salvadori, purement chimiques (Chemiker Zeitung, I90 I-9), sur la combustion de l'air dans la flamme de l'hydrogène, intéresseront les électrochimistes.

$$
* *
$$

On remarquera que l'auteur n'indique pas le rendement de ce procéde qu'il qualifie lui-même de remarquable. Estce oubli ou faute de renseignements ? D'autre part, cette seçonde disposition électrique que l'inventeur indique corrme étant plus propre à assurer une marche sans àcoup et dont l'auteur semble faire peu de cas en renonçant à la décrire, peut être intéressante à signaler. Nous reviendrons sur ce sujet et tiendrons nos lecteurs au courant des essais si intéressants qui se poursuivent un peu de toute part sur la fabrication électrochimique des nitrates.

Il est - soit dit en passant - regrettable de voir l'étranger aller plus vite que nous dans cette voie, alors que notre industrie hydro-électrique est pourtant la première intéressée à la solution de ce problème d'une importance capitale pour son développement.

\section{LE MOIS HYDRO-ÉLECTRIQUE en France et à l'Etranger}

\section{INFORMATIONS DIVERSES}

\section{Une circulaire ministérielle sur les formalités néces- saires à l'autorisation des Barrages.}

Avant de reproduire le texte de cette circulaire, qui est rédigée dans des termes que l'on aurait pu désirer plus clairs, sans mềme se montrer trop exigeant, nous croyons devoir en donner l'analyse.

On sait que l'article 12 de la loi du 8 avril 1898 , dans son 3 e paragraphe, a annoncé un règlement d'administration publique pour déterminer "la forme de l'instruction qui doit précéder les arrêtés * des préfets relatifs à l'établissement, dans les rivières non navigables, a d'ouvrages intéressant le régime ou le mode de l'écoulement des a eaux". Les autres ouvrages qui n'ont pas d'influence sur cet écoulement sont, en vertu de l'article ro de la même loi, dispensés de toute autorisation.

Le règlement annoncé n'a point encore paru; mais personne ne le réclame, car les demandes d'autorisation sont actuellement instruites dans les formes prescrites pour celles qui concernent les rivières classées, c'est-à-dire conformément à la circulaire, très complète et très claire, du 23 octobre I 851 . Toutefois le Ministre de l'Agriculture nous apprend que, le 2 t juin $\mathrm{I} \$ 99$, il a saisi le Conseil d'Etat de ce projet de règlement : La Haute Assemblée lui a répondu, que, pour elle, l'instruction faite dans les conditions ci-dessus indiquées est absolument suffisante, et que le besoin d'un nouveau texte ne se fait point sentir; qu'en tout cas ce règlement ne saurait être élaboré sans une étude préalable faite par les divers ministres compétents (Agriculture et Travaux Publics). Mais quelques chefs de service int, parait-il, exprimé au ministre leurs inquiétudes au sujet de certaines autorisations demandées pour l'établissement de "barrages sans revanche ", c'est-à-dire sans la protection de omi 6 en contrebas des rives. Le besoin d'avoir une force puissante pousse les industriels à créer des barrages beaucoup plus importants que ceux qui, autrefois, étaient destinés à la mise en marche de minoteries ou de scieries. Que faire en pareil cas ? C'est à cette question que répond la circulaire.

Tout d'abord le ministre ne parait pas espérer que la solution soit contenue dans le règlement qui sera élaboré par la Commission interministériclle; le nouveau texte, dit-il, tiendra peut-être ces questions à l'écart, et confiera à l'administration la solution de chaque espèce possible. Dans ces conditions, le service de l'Hydraulique agricole et, après lui, le ministre, a pensé que, sans attendre la décision interministérielle, il est préférable de réserver à l'Administration SUPÉRIEURE - lisez : le service Hydraulique du Ministère de l'Agriculture - un droit de contrôle sur les étáblissements importants, d'oú il résulte que le préfet, tout en restant théoriquement maitre de l'autorisation définitive, ne devra la donner qu'après avis du ministre. 
Quels sont les établissements importants visés par le ministre? La circulaire ne les indique pas. On ne saurait même déduire de son texte que tout barrage sans revanche doit être soumis au contrôle de l'autorité supérieure. Il est seulement prescrit à l'industriel de taire, dans ce cas une demande en deux expéditions, dont l'une doit être accompagnée de la description complète du barrage et de sa destination. Selon toute probabilité la nécessité de ces deux exemplaires est imposée pour permettre l'envoi de l'un des deux au ministre, si le préfet juge la demande suffisamment importante.

Mais,si le harrage proposé est destiné à une chute d'une puissance supérieure à cent poncelets, la demande devra toujours être soumise au ministre. Le dossier en entier devra lui être adressée, avec une étude faite conformément à la circulaire du 15 juin 1897 qui concerne les barrages-réservoirs.

Cette nouvelle circulaire n'est pas faite pour amener une plus grande rapidité dans les études préalables à l'autorisation, et elle est peu conforme aux idées dë décentralisation qui sont aujourd'hui prônées par tant de personnes compétentes !... Il est vrai qu'en guise de consolation, le ministre termine sa lettre en indiquant que les ingénieurs ne seront plus liés par le type usuel du règlement d'eau. Ils pourront se permettre quelques innovations, notamment en ce qui concerne lès clauses de déchéance pour non-usage.

On ne peut se défendre, cependaut, de cette pensée qu'étant sur place, habitués à la région pour laquelle le projet est établi, ils ont une autre compétence que l'administration centrale, non seulement pour édicter des déchéances, mais aussi pour imposer toutes mesures de précaution.

\section{Ministère de l'Agriculture, 9 septembre 1902.}

\section{Monsieur le préfet,}

Aux termes de l'article 12 de la loi du 8 avril 1898 , sur la "police des cours d'eau », les formes de l'instruction des demandes en autorisation d'établir des barrages sur les cours d'eau non navigables ni flottables doivent être déterminées par un règlement d'administration publique.

Le Conseil d'Etat, saisi parmoi, le 29 juin 1899 , de ce projet de règlement, a considéré que, d'áprès l'article 43 de la loi sus. visée, il devait être statué par des décrets rendus après instruction en ce qui concerne les demandes d'établissement d'ouvrages projetés sur les cours d'eau du domaine public et qu'antérieurement à la loi de 1898 , cette instruction avait lieu dans la même forme que lorsqu'il s'agissait d'autoriser un ouvrage sur un cours d'eau non navigable. La haute assemblée a jugé qu'il y avait intérêt à maintenir cette conformité dans tous les cas où aucune raison spéciale ne motive une différence de régime, et elle a subordonné son avis détinitif à l'étude de cette question par une commission interministérielle.

L'édiction du règlement se trouve donc momentanément ajournée.

D'autre part, sous l'impulsion des progrès de la science et du développement récent qui en est résulté pour les industries électriques, l'Administration se trouve placée en présence de questions nouvelles qui ne pouvaient être prévues à l’époque où fut élaboré le Code rural; il n'existait alors que des barrages destinés, pour la plupart, à la mise en marche de minoteries ou de scieries, et de dimensions assez modestes pour que les instructions générales du 23 octobre 185 I et du $26^{\circ}$ décembre 1884 restées en vigueur jusqu'à ce jour, aient pu imposer aux permissionnaires l'obligation de ménager, dans l'intérêt de la culture et notamment des prairies, une revanche de o, $6 \mathrm{~m}$ en contrebas des rives. Aujourd'hui, l'emploi de l'électricité pour l'éclairage des agglomérations urbaines, l'établissement des réseaux de tramways électriques, la fabrication en grand de l'aluminium et du carbure de calcium nécessitent la construction de barrages de dimensions telles qu'ils noient les rives et peuvent mettre en jeu la sécurité de toute une vallée.
Plusieurs chefs de services m'ayant consulté sur la procédure à suivre pour l'instruction des demandes en autorisation d'étabiir des barrages de cette catégorie, j'ai jugé opportun de généraliser les instructions qu'ils me demandaient et d'en faire l'objet de la présente circulaire, dont les prescriptions seront suivies jusqu'à ce que le règlement prévu par l'article 12 de la loi du 8 avril 1898 ait été publié. Au surplus, ce règlement ne pouvant outrepasser les limites de la délégation donnée par le législateur, il n'est pas assuré qu'il donne la solution des questions soulevées par MM. les Ingénieurs en chef; il laissera probablement à l'action administrative le soin de régler, par des mesures d'ordre intérieur, les questions qui sont du ressort de celle-ci, et, eu égard aux circonstances exposées plus haut, on peut se demander s'il ne serait pas opportun de le devancer par des instructions qui conserveront un caractère provisoire jusqu'à son édiction et resteront ensuite définitives en tout ce qu'elles n'auronit pas de contraire audit règlement.

C'est ce qu'a pensé la Commission de l'Hydraulique agricole que j'ai consultée à ce sujet. Elle a estimé qu'il ya un très grand intérêt à ce que les questions importantes que soulève la création des barrages sans revanche ne puissent être résolues sans que l'Administration supérieure ait été consultée et sans qu'elle ait pu s'assurer au préalable que la décision préfectorale à intervenir contiendra bien toutes les dispositions que l'Administration est en droit de prescrire pour la sauvegarde des intérêts généraux. La Commission a ajoụté que cette consultation, bien qu'elle ne soit pas prévue par la loi sur le régime des eaux, peut être rendue obligatoire par une instruction ministérielle, puisqu'elle ne constitue qu'une mesure administrative d'ordre intérieur et que le Ministre a toute qualité pour la prescrire.

J'ai, en conséquence, adopté, conformément à l'avis de la Commission, les dispositions suivantes:

I Toute demande ayant pour objet l'établissemeut d'un barrage sans revanche sur un cours d'eau non navigable ni flottable doit être adressée au Préfet en deux expéditions, dont une sur papier timbré, et doit être accompagnée, outre les renseignements dont la production est prescrite par l'instruction générale du 23 octobre $185 \mathrm{r}$, d'un projet complet du barrage et d'un mémoire dans lequel le pétitionnaire justifiera les dispositions qu'il propose et fera connaître le mode de fonctionnement de l'ouvragz, ainsi que les modifications que son établissement apportera au régime"des eaux. En attendant que le règlement d'administration publique prévu par l'article 12 de la loi du 8 avril 1898 ait été édicté, on continuera d'instruire les demandes dans les formes prescrites par les instructions générales des . 23 octobre 185 I et 26 décembre 1884 . Il sera statué par le Préfet;

$2^{\circ}$ Spécialement, lorsque l'usine projetée comportera une chute d'une puissance brute supérieure à cent poncelets, vous devrez, après la clôture de l'instruction et avant de prendre votre arrêté, me communiquer tout le dossier, de l'affaire, en y joignant, outre votre avis personnel, un rapport des ingénieurs contenant leurs propositions sur la suite à donner à la demande et une étude sur les conditions de stabilité de l'ouvrage projeté. Cette étude sera faite dans les formes prescrites par la circulaite du 5 juin 1897 relative à la révision des conditions de stabilité des barrages-réservoirs:

J'appelle à cette occasion, conformément à l'avis de la Commission, votre attention et celle de MM. les Ingénieurs sur ce que la clause de déchéance, inscrite dans le modèle de règlement d'eau annexé aux instructions générales susvisées, acquiert, lorsqu'il s'agit de grandes puissances hydrauliques, une importance particulière en ce qu’il est de nature à éviter les demandes dont l'objet effectif serait uniquement d'acquérir, sous le couvert 
d'un projet d'usine, des droits à indemnité à la charge des industriels qui viendraient ultérieurement s'établir dans la région. Au surplus, lorsqu'il s'agira de chutes de grande puissance, MM. les Ingénieurs ne devront pas se considérer comme liés d'une manière absolue et limitative par le type usuel de règlement d'eau. Tout en maintenant les clauses de style, ils devront y ajouter, dans chaquecas particulier, les dispositions qu'ils jugeront utiles pour préciser nettement les obligations du pétitionmaire et protéger efficacement les intérêts généraux dont l'Administration a la garde.

Recevez, etc.

\section{Le Ministre de l'Agriculture,} Signé: Movgeot.

N.-B. - Les différentes circulaires citées dans la lettre ministérielle du 9 septembre, sont, comme toutes celles qui concernent l'Hydraulique agricole, assez difficiles à trouver. La Revue est à la disposition de ses abonnés pour leur fournir tous documents et renseignements administratifs et techniques qui peuvent leur être utiles.

$$
\text { E.-F. CỗTE. }
$$

\section{A propos de la première expérience de transport de force.}

Sur la demande du Cercle des Anciens Elèves des Ecoles nationales $d^{\prime} A r t s$ et Métiers, nous insérons bien volontiers la lettre suivante datée d'Oullins (Rhône), le zo décembre rgoz:

Monsieur le Rédacteur en chef de La Honille Blanche, Lyon.

* Récemment abonnés à votre excellente revue, nous venons de parcourir avec beaucoup d'ntérêt les premiers numéros parus; nous les avons lus avec d'autant plus dattention que nous pensions $y$ trouver une rectification, en ce qui concerne la première cxpérience de transport de force par l'électricité.

"Dans le numéro I de La Houille Blanche, pages I et 2 , il est dit . Le jour où Marcel Déprez démontrait le principe de la réversibiluté des machines dynamo-électriques, c'est-à-dire réalisait le premier transport de force à distance, le problène de l'utilisation des chutes d'eau était du méme coup résolu dans toute son ampleur.

"Il y a là une erreur de fait que beaucoup de lecteurs auront sans doute rectifiée; mais il importe, néanmoins, de la relever, car c'est à Hippolyte Fontaine qu'est due incontestablement cette importante découverte - qui est en train de révolutionner l'industrie - et elle date de l'Exposition d: Vicnne, en 1873 , cest-à-dire bien avant les expériences retentissantes de Marcel Déprez.

" Dans le numéro de décembre ${ }_{1} 8_{7} 3$ de La Revue industriclle, pages 658 et 659 , sous le titre: * Les Machines Magnéto-Électriques A L'Expositron de VIENNE ", on peut lire ce qui suit : Les machines Gramme ont donné lien, à Viente, à une expérience des plus curieuses, qui pourra avoir un jour des applications très importantes. La machine principale, était actionnée par un moteur à gã Lenoir; l'électricité produite était envorée dans nue deuxieme machine Gramme de plus faibles dimensions, laquelle agissait en yéritable moteur électrique et actionnat une petite pompe centrifige Nett et Dumont. Conme les expérimentateurs n'avaient aucun appareil pour mesurer les forces dépensées et utilisées, et que, d'autre part, les machines exposées n'avaient pas été combines pour la transmission des forces, il na pas été possible de déterminer l'effet utile qui pourrait résulter d'une semblable installation. Cependant, ces premiers essais ont démontré non seulement la possibilité de transmettre une force à longue distance, mais ils ont encore fait yoir que le rendement etait notablement plus grand qu'avec l'emploi d'autres appareils, etc.

"Après cette citation, un peu longue peut être, il semble quil ne peut plus rester aucun doute sur la paternité de la découverte qui nous occupe et nous espérons que, dans l'intérêt de la vérité, vous voudrez bien insérer cette rectification dahs un prochain numéro de La Houlle Blanche.

"Veuillez bien agréer, Monsieur le Rédacteur en chef, mes respectueuses salutations".

Le pr.sident du Cercle:

L. CotTin.
Sans vouloir aucunement contester le mérite des expériences d'Hippolyce Fontaine, nous ferons observer que la question soulevée par nos correspondants comporte deus: points à élucider:

10 Qui a lepremier montré la réversibilité des michines à induction? Ne serait-ce pas Pacinotti lui-même? Les comptes rendus de l'Académie des Sciences doivent sans doute nous fixer sur ce premier point.

$2^{\circ}$ Quel est l'auteur de la première démonstration industrielle du transport de force à grande distance? En écrivant que c'est Marcel Déprez, nous avors voulu dire que ce sont bien les expériences de Vizille-Grenoble, en 1883 (, $r$ remier exemple de transport de force à 30 kilomètres) qui ont ouvert l'ère du transport électrique de l'énergie.

Et la Houille Blanche devra de transformer l'industrie moderne à trois hommes dont il convient d'unir les noms dans un même hommage d'admiration: Gramme, l'ınventeur, qui nuus a donné la machine dynamo, génératrice du courant électrique industriel; Marcel UÉprez, le savant, qui nous a montré le premier exemple de transport industriel de l'énergie par le courant électrique; Aristide Bergès, l'ingénieur, qui nous a enseigné l'art de capter le torrent et, par conséquent, de l'asservir à l'énergie sous toutes ses formes industrielles.

\section{L'Etat actuel de l'Industrie du Carbure de calcium et de l'Acétylène.}

Nous reproduisons ci-après la seconde partie de l'étude de M. Oscar Müusterberg, suivant la traduction de M. R. Chevassus, publiée par la Revue Générale des Sciences pures et appliquées (No du 15 juin 1902). Dans notre précédent numéro nous avons donné l'analyse de la première partie de cette étude. L'auteur examine, dans la seconde partie, la situation actuellement faite aux usines de carbure de calcium et ses considérations nous semblent devoir être citées in cxtenso.

"Tandis que pour l'utilisation de l'acétylène, dit-il, nous avons vu se développer une multitude de petits fabricants, et les grands capitaux rester complètement sur la réserve, nous constatons tout le contraire avec le carbure de calcium. Si l'acétylène n'a pu pénétrer dans la pratique par suite de l'incapacité de ses lanceurs et du manque de capitaux, la crise produite sur le marché du carbure a été amenée par les premiers industriels du monde, d spoiant des capitaux les plus forts qui aient peut-être jamais été mis au service dine entreprise industrielle. L'industrie du carbure est comme sacritiée aux conditions malsaines qui furent la conséquence du développement exagéré des grandes sociétés d'électricité en ces dernières années. Ce n'est pas ici le lieu de rechercher de plus près l'influence exercée sur l'industrie allemande par la diffusion des affaires financières et des dépôts dans les banques, pas plus que par la transformation des directeurs de grandes usines en financiers. Le principe de tinancialisation, appliqué avec une extrême prudence, peut bien conduire à un succès malgré les écueils qu'il cache, comme le prouve le brlllant développement d'entreprises industrielles particulières, mais le principe lui-même n'en est pas moins mauvais en général. Quand les honnes affaires sont épuisées, on cherchẹ de nouvelles occupations aux usines toujours plus grandes, pour ne pas avouer une réduction du personnel, et on accepte des entreprises auxquelles manque une base solide. Les établissements de banque ne sont presque jamais en mesure de connaitre l'importance technique de leurs entreprises industrielles, et s'abandonnent à leurs ingénieuts-conseils, lesquels, tout en différant souvent d'avis, finissent cependant toujours par décider, en dernier ressort, dans le groupe financier. L'ingénieur est tout heureux de sentir les ri-ques passer de ses épaules aux mains de banques puissantes et du public qui, derrière celles-ci, est tout plein d'une aveugle confiance pour la finance et l'industrie, et il entreprend, pour le compte d'autrui, des affaires dont il ne voudrait pas pour lui-même. L'intérêt primordial de l'industriel est de se créer, dans le monde, une clientele de succursales pour ses machines, ses brevets et ses proc.dés 11 ne s'inquiète pas de savoir si toutes les conditions destinées à en asiurer l'existence sont bien réalisées. Puis, quand ces filiales viennent à péricliter, c'est la faute de leurs ałministrateurs particuliers ou de circonstances toutes spéciales, et non pas celle des parents de cet enfant, hystérique déjà à sa naissance. 
"Ce fut un événement considérable pour toutes les branches de lindustrie que la découverte du carbure de calcium, adopté ensuite par l'Electrochimie comme un produit de grand avenir, ouvrant encore de nouveaux domaines à l'activité des électriciens. Tout d'abord on garda le plus grand secret sur la fabrication, et les rares usines qui s'en occupèrent durent payer cher leurs essals. Le public découvrit rrop tard que la fabrication était bien plus simple qu'on le disait. Tandis que les uns sacrifiaient, pour ces prétendus secrets, une partie de leurs capitaux, d'autres, qui les possédaient, laissant tout cela, expérimentaient par eux-mêmes et réussissaient mieux encore.

"La déchéance du brevet Bullier fut un grand malheur pour la jeune industrie. S'il était resté en vigueur, un petit nombre de fabriques auraient seules pu se monter et faire de bonnes affaires avec un prix modique pour la vente. Les vainqueurs, dans ce tournoi, seraient maintenant plus riches de plusieurs millions, s'ils eussent alors succombé!

"Une fois le brevet tombé, ce fut, dans toute l'Europe, une course folle à la recherche des chures d'eau. Au fond du nord de la Suède et de la Finlande, au milieu des montagnes de la Savoie et de la Suisse, en Bosnie même, on achetait les chutes géantes pour bâtir là-dessus des sociétés par actions. Les prospectus montrent encore quelle ignorance présidait à ces achats : l'argent s'offrait partout à bon compte; l'électricité nous avait présenté tant de merveilles qu'on pouvait apercevoir la perspective daffaires extraordinaires dans les brillants avantages du nouvel éclairage. Les possesseurs de chutes, anguère reléguées aux limites du monde civilisé, crovaient se découvrir des trésors cachés et faisaient tous leurs efforts pour les convertir sinon en or, au moins en papier. Ce mouvement était encouragé surtout par les Sociétés de constructions électriques, désireuses de se créer des débouchés pour leurs machines. C'est ainsi qu'une seule maison a fait payer cinq millions de francs l'installation de 8.000 chevaux électriques à l'usine de Jaîce, $\in n$ Bosnie, et le mal ne faisait que s'accroitre par l'engoueñent du public moutonnier.

"Ensuite, chaque usine se montait en secret, sans même discuter ses plans avec les gens du métier et, quand toutes ces usines apparurent ensemble sur le marché, voici ce qu'elles purent y voir : l'Amerique surproduisait déà et exportait même; la France couvrait largement sa propre consommation; enfin, en Allemazne, une seule grande fabrique aurait pu suffire aux besoins. Les énormes stocks offerts amenèrent inévitablement la crise que rendaient encore plus aigue les difficultés financières dans lesquelles ces usines se débattaient presque toutes.

« D'ailleurs, la pureté du produit en question ne dépendait pas des procédés, brevetés ou non, mais uniquement du choix des matières premières. Il y avait tien plus lieu de se soucier des frais et des conditions réelies de la production en grand, ainsi que des facteurs, variables avec les localités, relatifs à la proximité des matières premières et à la facilité de se procurer la main-d'œuvre, et de rúexpédier le produit manufacturé. De là, des différences énormes dans les prix de revient, d'une part, puis dans les prix de vente. Il faut aussi aux usines certaines conditions générales pour assurer leur existence, et une fabrication de moins de 2.000 tonnes me parait manquer absolument au but qu'on se propose; il faudra aussi toujours chercher à utiliser le maximum d'énergie, et les usines à fabrication modique ou à faible débit ne pourront subsister.

"La formation du Syndicat à la suite de la crise a eu pour effet, sinon de relever énergiquement les prix gâtés par quelques dissidents, tout au moins de mettre en évidence cette énorme surproduction et d'arrêter la construction de nouvelles usines. Mais la lutte pour la vie n'est pas supprimée, et toute une série d'usines sans grands capitaux devra disparaitre ou se vouer à une industrie plus rémunératrice. Et le marché du monde, à l'exception de l'Amérique et de la France, protégées par leur particularisme national, sera tenu par quelques fabriques à puissants capitaux.

" En tout cas, le public peut compter que les prix ne remonteront pas notablement. Les usines ont le plus grand intérêt à les maintenir assez bas pour s'assurer une clientèle suffisante. Ce résultat une fois acquis, elles devront, pour augmenter leurs bénéfices, non pas relever les prix, ce qui arrêterait la consommation et troublerait l'industrie, mais chercher plutôt à se créer encore plus de clients. Aussi les fabricants d'appareils à acétylène et le public peuvent-ils assister tranquillement à la lutte des usines à carbure qui leur assure le bon marché de ce produit.

* L'anné prochaine nous apprendra lesquelles de ces usines auront la force de résister. Pour être en nesure de vivre, il leur faudra pouvoir employer l'énergie et le personnel à une autre fabrication, pendant les temps d'arrêt, mais je crains encore de nouveaux désastres.

" Je termine en affirmant encore que l'acetylène est l'éclairage de l'avenir dans les petités villes et les édifices isolés. Les causes de la crise présente sont la surabondance des capitaux pour le carbure de calcium et leur rareté pour l'acétyline; ensuite, le manque d'entente pour la division du travail entre le fabricant d'appareils et l'installateur. Quand tout cela aura changé, la propagation se fera bien vite, ct les sommes considérables sacritiées dans notre apprentissage auront servi à assurer à l'acétylène le développement sain et normal anquel il a aroit $n$.

Quelle psychologie de la science des affaires industrielles!..

N'était-il pas interessant de reproduire ici cet exposé si juste de la situation économique de l'industrie du carbure de calcium? Les considérations de l'ancien directeur de l" allgemeine Carbid und Acetylen Gesellschaft "s'appliquent d'une façon particulièrement frappante à lévolution de cette industrie en France.

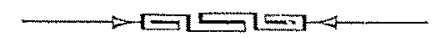

\section{ACADÉMIE DES SCIENCES}

\section{Séance du 3 novembre 1902.}

Sur la résistance électrique du sulfure du plomb aux très basses températures. - Note de M. Edmond van Aubet.

L'auteur a fait des expériences sur une tige de sulfure de plomb en coulant le produit pur fondu dans une lingotière cylindrique au préalable fortement chauffée. Cette tige était ensuite limée et usce avec précaution, de manière à réaliser un cylindre qui avait $5 \mathrm{~m} / \mathrm{m}_{9}$ de diamètre. Cette petite baguette était absolument massive et ne présentait aucune soufflure. Elle étnit munie à ses extrémités de pinces en laiton permettant de mesurer sa résistance électrique par la méthode de lord Kelvin.

La résistivité du sulfure de plomb pur et coulé, a été trouvée de 289,88 microhms-centimètres à la température de $+20^{\circ}, 7 \mathrm{C}$. Cette résistivité diminue toujours à mesure que la température s'abaisse, en sorte que, dans l'air liquide, la résistance de la tige est inférieure au $1 / 4$ de sa valeur à la température précédente.

Le sulfure de plomb coulé se comporte donc, entre les limites de température considérées, comme les métaux purs et sa résistivité électrique est considérablement plus faible que celle de la pyrite naturelle $\mathrm{FeS}^{2}$, dont la résistance diminue quand la température s'élève. Si l'on trace la courbe qui exprime la variation de résistance. électrique avec la température, on constate qu'elle ne présente pas une forte courbure.

\section{Séance du lo novembre rgoz.}

Sur la conductibilité des dissolutions aux basses températures. - Note de M. J. Kunz.

L'affaiblissement considérable de la conductibilité électrolytique: aux basses températures peut provenir : soit de l'abaissement du degré de dissociation, soit de la viscosité croissante que le milieu. oppose aux ions.

M. Kunz a procédé à des mesures de conductibilités électrolytiques. à des températures aussi basses que possible, mais en restreignant ses recherches aux dissolutions concentrées, à point de congelation. très bas, d'acide sulfurique, de soude caustique et de chlorure de- 
calcium. Il a pu atteindre, avec les solutions d'acide sulfurique de de 45 à $70 \% / \%$ des températures inférieures à $-70^{\circ}$.

Les résistances ont été déterminées par la méthode de Kohlrausch, avec un pont à fil exactement calibré. Les températures jusqu'à - $35^{\circ}$, ont été mesurées avec un thermomètre norazal de Pernet, et, au-dessous au moyen du couple constantan-fer, préalablement étalonné.

Les courbes représentant la conductibilité en fonction de la température ont une similiture d'allure corroborant l'idée qui est à la base de cette hypothèsé, à savoir que la cause principale de la radiation thermique réside dans la yiscosité du milivu pour les ions.

Ces expériences montrent aussi bien clairement le contraste des propriétés des électrolytes et des métaux. Tandis que la résistance de ceux-ci s'annule au zéro absolu, c'est la conductibilité des électrolytes qui semble y tendre vers une valeur nulle.

Nouvelles expériences sur la résistance électrique du sélénium et ses applications à la transmission des images et des impressions lumineuses. - Note de M. Dussaud.

Depuis longtemps l'auteur s'occupe de cette question et il réalise ses expérnences au moyen de deux postes reliés par un courant électrique.

"Le poste transmetteur se compose d'une surface plane, non conductrice, divisée par de légères cloisons en carrés égaux d'environ 5 centimetres de côté; dans chacun d'eux est disposée une bobine, formée d'une lame en matière isolante, sur laquelle sont enroulés deux fils de cuivre de petit diamètre, noyés lans une couche de sélénium préparée de manière à lui assurer le maximum de sensibilité, en me basant sur mes expériences antérieures. Un de ces fils est parcouru par un faible courant électrique d'ordre téléphonique.

"Lorsqu'on éclaire la couche de sélénium, celle-ci acquiert une conductibilité d'autant plus gran'de que l'éclairage est plus intense et laisse passer une partie du courant dans le second fil.

"Ce dernier est relié à une bobine munie d'un contact, faisant l'office d'un servo-moteur, destiné à agir sur un courant local d'une énergie suffisante pour allumer des lampes à incandescence au poste récepteur. qui se compose, ainsi que le poste transmetteur, d'une surface plane, divisée aussi par des cloisons en un mème nombre de cellules carrées contenant chacune une lampe à incandescence.

"Lorsqu'au poste transmetteur on éclaire une ou plusieurs bobines recouvertes de sélénium, la conductibilité qui se développe permet à un faible courant de traverser le second fil relié au servo-moteur; on voit alors s'íclairer, au poste d'arrivée, les lampes correspondantes à celles trappées par la lumière au poste de départ $x$.

L'auteur conclut en terminant que, d'après ses expériences, il sera possible de transmettre des impressions lumineuses et des images à de grandes distances.

\section{Sur le fonctionnement et l'alimentation de la fontaine de} Vaucluse. - Note de M. E.-A. Martel.

«Il est généralement admis que Vaucluse constitue le trop plein d'une vaste nappe d'eau souterraine, formant une réserve d'au moins io millions de mètres cubes.

"Or, Vaucluse est simplement le débouché dun fleuve souterram, dont l'écoulement a lieu par de longs et hauts canaux renflés au milieu, où de considérables variations de niveau se produisent sous la double influence : $1^{\circ} \mathrm{du}$ jeu rígulier des précipitations atmosphériques et des infiltrations qui en résultent ; $2^{\circ}$ des rétrécissements, siphonnements et éboulements intérieurs, agissant comme des vannes retardatrices et transformant ces canaux en réservoirs temporaires étroits. Ces variations, en engendrant de grandes différencés de pression hydrostatique, provoquent une mise en charge plus ou moins considérable des veines liquides ramifiées sous terre; et la répercussion de cette pression variable sur le dernier vase communicant qui forme, dans une faille, l'émergence de Vaucluse amène les écarts de niveau et de debit de la fontaine $s$.

M. Martel schématise sur une planche jointe au texte de sa note, lallure et la disposition du systène hydraulique de Vaucluse. Setles quelques-unes des coupes d'avens y correspondent à la réalité des choses matériellement constatées, dit-il ; mais le surplus des profils ne fait que reproduire des formes expérimentalement relevées ailleurs.

"Cette synthèse n'est donc théorique que pour une partie. Et, si lon entreprend jamais le colossal travail de la désobstruction des avens, pour parvenir aux collecteurs de Vaucluse, on leur rencontrera certainement des coupes et des profils analogues.

" Mon hypothèse, d'ailleurs, satisfait pleinement aux données recuellies depuis 1873 , par la commission météorologique de Vaucluse.

" $1^{\circ}$ La cavité la plus proche de lémergence n'est pas d'une grande capacite à cause des particularités météorologiques constatées : d'une part, en effet, la température moyennne annuelle de la fontaine s'est toujours montrée de $2^{\circ}$ inférieure à celle de lair ; d'autre part, la température de l'eau, chaque année, varie en moyenne de y $^{0}$ 5. Donc, jusqu'au voisinage immédiat de l'émergence, le courant souterrain se manifeste, et larrivée des eaux froides, descendues des hauts plateaux du Ventoux, de Lure, etc., empêche la fontaine d'équilibrer sa température avec celle de l'air extérieur et d'acquérir la stabilité thermique que devrait lui communiquer un réservoir vaste et profond. Au surplus, il est établi que, dans les années les moins pluvieuses, les variations de température sont les plus faibles.

" $2^{\circ}$ Les caprices du débit (minimum : $4^{\mathrm{m}^{3}}$ par seconde; maximum : $150 \mathrm{~m}^{3}$ par seconde), sont expliqués par les étranglements et siphonnements qui provoquent les mises en charge, après les pluies, et ralentissent l'écoulerent lors des sécheresses pour assurer un étiage rarement inférieur à 6 ou $\mathrm{S}^{3}$.

" 30 Après les pluies abondantes et longues, le débit de la source ne diminue que très lentement, grâce à ce retard dans la vidange des collecteurs.

" $4^{\circ}$ Les pluies des régions yoisines de la source se manifestent plus rapidement que celles des localités eloignées, parce que leur trajet souterrain est moins long et entravé par moins d'obstacles...

" 50 Les pluies influent lentement sur le debit de la fontaine quand elle est trés basse, parce que la partie inférieure et très étroite des collecteurs est seule remplie à l'étiage, et qu'il faut aux iufiltrations le temps de s'élever dans le renflement de la partie moyenne.

" $6^{\circ}$ La fontaine grossit rapidentent dès qu'elle atteint une certaine hauteur, parce que le remplissage du renflement de la partie moyenne augmente la mise en charge et accroit promptementle débit de l'émergence.

" $7^{\circ}$ Les pluies fortes et prolongées font croître liz source également à cause de l'élévation du niveau et de l'augmentation de pression hydrostatique.

"Ainsi toutes ces manifestations s'expliquent très facilement en: appliquant au résean hydrologique souterrain de Vaucluse les configurations de courants intérieurs, dont les exemples se multiplient chaque année parmi les régions calcaires, avec une similitude de plus en plus générale.

"Et il convient de conclure à l'abandon définitif, en matière d'alimentation de sources, du terme incohérent et fautif de nappe valiclusienne; l'usage d'une association de mots aussi peu conforme à des lois naturelles, maintenant dûment établies, ne peut que conduire les géologues à de flagrantes erreurs, et les hydrologues à de fâcheux $x$ micomptes.

\section{Séance du I7 nonembre I902}

\section{Sur les électrodes bipolaires. - Note de MM. André Bro- Chet et C. -L. Barillet.}

Les auteurs ont étudié comment se comporte une électrode bipolaire lorsqu'elle ne forme pas cloison étanche et, d'une façon générale l'influence d'une masse métallique placée dans un électrolyseur. et ne communiquant pas avec les électrodes.

Ils ont choisi, pour électrolyte, le sulfate de cuivre, et se sont servi d'une électrode bipoláire en platine, l'interélectrode, le côté de cette lame en regard de l'anode étant l'intercathode, le côté face à la cathode, l'interanode.

L'augmenfation de poids de la lame: de platine correspondant au 
cuivre déposé sur l'intercathode permet d'évaluer la quantité d'électricité ayant traversé cette lame.

Pour une surface déterminée de l'interélectrode en rapport avecles constantes de l'électrolyseur, on remarque qu'll ne se forme, au-dessous d'unecertaineciensité de courant, aucun dépot sur la lame de platine, celle-ci se comportant comme une lame non conductrice et n'ayant d'autre résultat que d'augmenter la résistance ohmique du bain et la tension aux bornes de l'électrolyseur. Mais à partir d'une densité de courant déterminée, correspondant dans tous les cas à une différence de potentiel aux bornes supérieure à la tension de décomposition du sulfate de cuivre, une certaine quantité d'électricité traverse la lame de platine, ce qui est démontré par le dépôt de cuivre sur l'interanode et un dégagement gazeux sur l'intercathode.

Ce dépôt de cuivre affecte des contzurs variables suivant la déformation du flux de courant produite par l'électrode bipolaire, mais dans leurs expériences les auteurs ont toujours eu un cercle au centre de l'intercathode.

Il ne suffit pas que la différence de potentiel aux bornes soit supérieure à la tension de décomposition pour que le courant traverse la bipolaire, il faut encore que le rapport des surfaces soit assez élevé.

Les auteurs ont réuni dans un tableau les dimensions du cercle de cuivre déposé sur l'intercathode, correspondantes aux diverses tensions aux bornes de l'électrolyseur et avec des écarts de $4^{\mathrm{cm}}, 8 \mathrm{~cm}$, r $2^{\mathrm{em}}$ entre des électrodes de $13 \mathrm{~cm}$ j de côté.

Il ressort de ce tableau que la quantité d'électricité qui a traversé l'interélectrode a toujours été très faible, et n'a jamais dépassé, dans les meilleures conditions, $2,5 \%$ du courant total fourni à l'appareil.

Les auteurs passent ensuite brièvement en revue les divers points suivants:

$1^{\circ} \mathrm{Si}$, au lieu d'une électrode bipolaire, on en met deux, à densité de courant égale, la tension est plus élevée et le dépôt plus faible;

$2^{\circ}$ Si l'on déplace une électrode bipolaire, soit vers l'anode, soit vers la cathode, on constate que la tension aux bornes diminue;

3. Si l'on déplace une électrode bipolaire, en maintenant l'intensité constante, on remarque que la surface du dépôt augmente et que son épaisseur diminue, si on la rapproche de l'anode; au contraire, la surface du dépôt diminue, mais son épaisseur augmente, si on la rapproche de la cathode;

$4^{\circ}$ L'interélectrode étant une lame de platine dont le côté anode a été recouvert préalablement d'une couche de cuivre, l'interanode agissant comme électrode soluble, il se forme sur l'intercathode, un dépôt sensiblement uniforme; puis, lorsque le métal anodique a complètement disparu, la tension aux bornes s'élève presque instantanément et le cuivre déposé sur les bords de l'intercathode se dissout peu à peu; finalement, il reste sur l'intercathode un cercle plus grand que celui obtenu par dépôt direct. Entre le cercle ainsi rongé et le cercle formé par le dépôt pendant la marche régulière, se trouve un anneau correspondant à une zone neutre;

50 Cet essai étant reproduit dans des conditions telles que la tension aux bornes soit inférieure à la tension de décomposition du sulfate de cuivre, le même fait se produit th le cuivre s'accumule au centre de l'intercathode; puis l'action s'artête, le poids de la lame restant invariable pendant la durée de l'expérience.

Sur la constante de temps caractéristique de la disparition de la radioactivité induite par le radium dans une enceinte fermée. - Note de M. P. Curie.

L'auteur examine la manière dont disparait la radioactivité induite quand on a supprimé l'action du radium. Il ne s'occupe que de la loi de désactivation dáns le cas d'une enceinte close, renfermant des gaz activés. Il emploie, comme enceinte close, un tube de verre scellé à la lampe, lequel est placé dans le cylindre intérieur d'un condensateur cylindrique en aluminium. Les rayons émis par le tube traversent l'aluminium et rendent conducteur l'air entre les armatures du condensateur. On mesure le courant limite que l'on obtient entre les deux armatures, lorsqu'on maintient entre elles une différence de potentiel suffisante ( 450 volts). Le rayonnement ainsi mesuré est dû exclusivement à la radioactivité des parois, car, lorsque l'on retire rapidement l'air actif du tube, le rayonnement, mesuré immédiatement après, est le' même qu'avant.
La loi de désactivation d'une enceinte fermée est remarquablement simple. L.'intensité du rayonnement s'exprime en fonstion du temps par une loi exponentielle. Dans la formule entre une constante de temps qui caractérise la diminution de l'activité de l'enceinte. Cette constante, d'après les expériences de l'auteur faites dans des conditions extrêmement variées, n'est nullement influencée par les conditions de l'expérience, par la nature du gaz qui remplit l'enceinte ou de la matière qui en constitue les parois; elle ne comporte donc aucun caractère spécifique et doit avoir une importance d'ordre général. M. Curie estime qu'elle peut être déterminée avec unegrande précision.

\section{SOCIÉTÉ INTERNATIONALE DES ÉLECTRICIENS}

\author{
Bulletin de novembre 1902 .
}

\section{Application de la méthode de la boucle à la recherche d'un défaut dans un cable triphasé souterrain.}

M. P. JANET décrit la modification ingenieusement apportée à la méthode classique par $M$. Iliovici, attaché au Laboratoire central.

Lorsqu'on applique la méthode, bien connue, de la boucle à la localisation d'un défaut dans le cas des câbles à fortes sections, on est quelquefois gêné par la résistance des fils de secours et des contacts qui reuvent apporter des erreurs notables dans les résultats de la mesure. M. Iliovici a profité de ce que, dans le cas dont il. avait à s'occuper, deux des conducteurs sur trois étaient intacts, pour éviter cet inconvénient.

Il a éliminé l'effet des contacts et des fils de connexion en les faisant entrer dans la formation des bras d'un pont de Wheatstone contenant de très grandes résistances. Les deux câbles intacts de la canalisation à examiner étaient employés comme fils de connexion.

\section{Sur les mesures magnétiques industrielles.}

M. Armagnat fait un exposé critique des méthodes et des appareils industriels pour les mesures magnétiques.

Les grandeurs que l'on a à mesurer sont : linduction $\mathcal{B}$; le champ magnétique $\mathfrak{H} \mathcal{C}$ et la perméabilité $\boldsymbol{\mu}$. Ces facteurs n'ont isolément aucun inrérêt, et il faut toujours considérer en mème temps deux d'entre eux : $\mathcal{B}=f(\mathscr{H} \mathcal{C}), \mu=\varphi(\mathscr{H}), \mathcal{H}=\Psi(\boldsymbol{\mu})$. Les fonctions $f$, $९$ et $\Psi$ sont mathématiquement inconnues; il faut les déterminer expérimentalement.

La courbe complète d'un cycle magnétique ne peut pas servirà caractériser les qualités magnétiques d'un fer; c'est la courbe de la perméabilité qui doit servir à caractériser les grandeurs précédemment considérées.

L'auteur, examinant les restrictions que l'on peut faire à la Loi de Steinmetz, conclut qu'étant donné le peu de régularité des tôles du commerce, cette loi peut néanmoins rendre de grands services. Il fair ensuite observer que, lorsque le circuit magnétique n'est pas homogène, ce qui est le cas des perméamètres industriels, il faut tenir compte des parties étrangères à l'échantillon mesuré et que la correction à faire comporte un certain aléa dû aux facteurs inconnus ou impossible à mesurer exactement.

La fonction qui relie $\mathcal{B}$ et $\mathcal{H} \mathcal{C}$ n'ayant pas d'expression mathematique connue, il importe de réprésenter graphiquement les résultats d'expérience, et en même temps de tracer les courbes $\mathfrak{B}=\Psi(\boldsymbol{\mu})$ et $\boldsymbol{\mu}=\varphi(\mathfrak{H}) \cdot$. Ces deux dernières courbes ne sont nullement caractéristiques du fer essayé, mais elles permettent, plus sûrement, la comparaison des méthodes ou des appareils. Ft des considérations qui précèdent, M. Armagnat déduit la définition de la tolérance a accorder dans les conditions d'un marché. Cette tolérance sera bien défnie en disant que les échantillons essayés devront donner la courbe $\mathcal{B}=f(\mathfrak{J} \mathcal{C})$ adoptée, avec une tolérance de $n \%$ sur $\mathfrak{T C}$ et de $m_{0} /{ }^{\circ}$ sur $\mathcal{B}$ puis examinant comment se traduit le terme de correction, il conclut à l'énoncé de la règle suivante : "Dans tous les les appareils où les joints modifient la valeur de $\mathfrak{H} \dot{C}$, il faut faire et 
défaire les joints plusieurs tois de suite, avant la mesure, et s'arrêter à la position qui, pour $\mathfrak{H} \mathcal{l}$ constant, c'est-à-dire pour une intensité de courant constante, donne la valeur la plus élevée de $\mathcal{B}$."

Enfin, avant de passer à l'examen des méthodes et appareils, lauteur indique les précautions à prendre, tant dans la préparation des échantillons que dans le traitement magnétique qu'on leur fait subir, pour que les mesures magnétiques aient une valeur réelle.

Méthode balistique. - En principe, elle consiste à mesurer la quantité d'électricité induite par une variation brusque du courant magnétisant, dans une bobine enroulée sur le fer étudié. 11 faut que l'échantillon ait la forme d'un tore ou celle d'un barreau droit à grande longueur. Avec le tore linconvénient est de faire des enroulements bien réguliers, des bobines magnétisante et induite, à chaque échantillon. Avec un harreau droit, il est facile de construire des bobines, une fois pour toutes, qui contiendront exactement les échntillons, mais comme on est obligé de donner au barreau une longueur de 300 à 500 fols son diamètre, on est conduit à des dimensions peu pratiques.

Appareil d'Hopkinson. - Il a pour but, en employant des bobines faites une fois pour toutes, d'opérer sur des échantillons de dimensions plus pratiques. 11 se compose d'un gros bloc de ter percé d'une ouverture rectangulaire dans laquelle sont logées, suivant un axe longitudinal, deux bobines magnétisantes ayant entre elles la bobine induite; le barreau de fer à étudier est introduit dans l'axe de ces bobines. On tire le meilleur parti de l'appareil en laissant la bobine induite fixe et en procedant par variation de courant. Pour connaître $\mathscr{H} \mathcal{C}$, il faut diviser les ampères-tours par la longueur effeclive du barreau et faire la correction relative à la réluctance du bloc de fer, difficulté qui conduit à déterminer la correction expérimentalement à l'arde d'un barreau étudié par d'autres moyens.

Méthode d'Euving. - Elle consiste à étudier ensemble deux barreaux identiques que l'on place dans des bobines magnétisantes et que l'on réunit par deux jougs en fer bien ajustés. L es bobines magnétisantes remplissant exactement l'espace entre les jougs, et une bobine induite étant placée surl'un des harreaux, on mesure l'induction $\mathcal{B}$ qui correspond à diffirentes valeurs du courant magnétisant. Cette méthode ne donne des résultats exacts que lorsque l'ajustage des jougs a été parfaitement exécuté.

Perméanètre de Drysdale. - Le but de cet appareil est d'étudier la qualité du fer sur la pièce essayée elle-même, une carcasse de dynamo par exemp.e. A cet effet, $M$. Drysdale, perce à l'aide d'une fraise annulaire spéciale, un trou dans une partie de la pièce ou il ne cause pas de détérioration; il a gmm 5 de diamètre et 16 millimètres environ de profondeur, tandis que la tige cylindrique réservée au centre a $2^{\mathrm{mm}} 5$ de diametre. Un bouchon en fer doux, portant une bobine magnétisante, vient alors s'adapter à ce trou de telle façon que la tige centrale forme le noyau de la bobine. L'ensemble constitue un perméamètre analogue à celui d'Hopkinson, dans lequel l'échantillon a une longueur effective de $12 \mathrm{~mm} 7$. C'est cette faible longueur qui constitue le défaut de l'appareil.

Pont magnétique d'Ewing. - Son principe est le suivant: on compare deux barreaux identiques comme dimensions, chacun étant placé dans une boline magnétisante; leurs extrémités sont engagées dans des culasses de fer, de grande section et de faibles longueur. Lorsque la force magnétomotrice, dans craque bobine, est proportionnelle à la réluctance de la barre correspondante, les deux culasses de fer şont au même potentiel magnétique et le rapport des ampèrestours dans les deux bobines donne le rapport des réluctances. La mesure finie, et le courant magnétisant observé, il suffit de se reporter à la courbe fournie avec le barreau étalon.

Perméanetre Lamb et Walker. - Cet appareil se rattache aux ponts magnétisants par son principe qui consiste à égaler la réluctance de l'échantillon à celle d'un entrefer de longueur variable et de section croissante. Le fer étudié, entouré d'une bobine magnétisante, est appuyé par un joug contre une culasse devant laquelle se meut un piston, un entrefer qui existe entre ces deux pièces complétant le circuit; une bobine magnétisante entoure cette dernière partie de l'appareil. Les mesures se font par réglage de l'entrefer.
Balance d'Eving. - Elle utilise la force portante des aimants pour la mesure de $\mathcal{B}$. L'échantillon, sous forme de baguette cylindrique, est placé à l'extrémité d'une règle divisée, sur laquelle glisse un poids curseur; l'un des bouts de l'échantillon s'appuie dans une encoche, à la joue d'un électro-aimant, dans lequel passe le courant magnétisant; l'autre bout ne peut avoir qu'un point de contact avec l'autre joue. La mesure se fait en cherchant la position du curseur pour laquelle l'attraction magnétique est insulfisante à maintenir ce dernier contact.

Balance magnélique de Du Bois. - Ici, les attractions à distance sont substituées a la force portante. L'échantillon est placé dans une bobine magnétisante horizontale, terminée à ses deux extrémités par des épanouissements polaires en acier. Au-dessus, oscille un fléau massif à bras inégaux et ne pouvant jamais avoir de contact avec les épanouissements polaires. Quand la bobine magnétisante est excitée, les attractions égales de chaque côté, déterminent des moments inégaux. On rétablit l'équilibre au moyen de curseurs glissant sur des règles graduées, et la valeur de $\mathcal{B}$ est proportionnelle à la racine carrée du déplacement des curseurs.

Perméamètre de Brilger. - On sait que la résistance d'une spirale de bismuth va:ie rapidement quand le champ magnétique dans lequel elle se trouve augmente; on introduit une spirale de bismuth entre les deux parties égales de l'échantillon coupé en deux et placé dans une bobine magnétisante, et c'est en mesurant la variation relative de cette résistance, que lon déterminc $\mathcal{B}$; une courbe d'étalonage donne la relation entre ces deux quantités.

Perméametre de Kopsel. - Cet appareil constitue une sorte de galvanomètre Déprez-d'Arsonval, dans lequel le champ magnétique est produit par le flux de force développé dans le ter étudié.

Perméamétre de Carpentier (rgoo). - Un tore à section rectangulaire, situé dans un plan horizontal, est coupé, suivant un diamètre, de deux entrefers égaux. Dans l'un, est logée une petite aiguille aimantée dont la position normale est perpendiculaire aux lignes de forces du flux mesuré; elle est suspendue par un fil à un bouton moleté portant deux index qui se déplacent devant les divisions d'un cadran à la partie supérieure du tube de suspension du système. Le barreau essayé est placé dans la bobine magnétisante suivant un diamètre perpendiculaire à celui qui joint les entrefers. Les joints sont rendus aussi parfaits que possible.

Le flux de force mesuré fait dévier l'aiguille; on la ramène à sa position normale en tournant le bouton; puis on fait tourner le cadran de facon à placer les index aux zéros des graduations. Ensuite, renversant le courant, on ramène l'aiguille à sa position normale; à ce moment, les deux index indiquent l'un $\mathcal{B}$ et l'autre la correction.

L'auteur décrit ensuite le perméamètre G. Baily quí mesure directement la perméabilité en employant deux bobines magnétisantes parallèles, recevant chacune un échantullon, et dont les pièces polaires, à l'une de leurs extrémités, exercent une action magnétique sur un équipage astatique placé au-dessus d'elles; puis il arrive au :

Perméamètre Picou. - Cet appareil a pour but d'éliminer l'effet des joints.

Le barreau à essayer est placé entre deux blocs de fer égaux possédantdes bobines d'abord réunies de telle sorte que ces blocs soient, magnétiquement, en série. Le barreau n'est alors parcouru par aucun Alux longitudinal. Si, ensuite, on renverse le sens du courant dans l'une des précédentes bobines et que l'on envoie un courant de sens convenable dans la bobine entourant le barreau d'essai, il est possible de régler ce courant de telle sorte que le flux, dans les blocs considérés, reprenne la valeur qu'il avait dans le premier montage. Pour. le détail des opérations nous renvojons au mémoire de l'auteur. Cet appareil a le mérite de seprêter indifféremment à l'essai desbarreaux pleins et des faisceaux de tòles, pour lesquels il n'exige qu'un ajustage très grossier.

Mesure do l'hystérésis. - Il n'existeguère que deux appareils pratiques pour la mesure de l'hystérésis; aussi a-t-on souvent recours à la méthode indirecte qui consiste à tracer le cycle complet, par la méthode balistique ou à l'aide de perméamètres, puis à mesurer la surface de ce cycle.

M. Armagnat indique deux procédés permettant de mesurer l'hys- 
térésis, pour un cycle donné, à l'aide d'une seule observation, et dit: "Quand on passe de ces máthodes à l'emploi des hystérésimètres, la simplification est grande et il faut reconnaitre que, même encore plus grossiers, ces appareils rendraient da réels services, le manque d'homogénéité des fers du commerce rendant illusoires les essais précis.» Suit alors la descrintion sommaire des hystẹrésimètres d'Ewing et de Blondel basés sur ce principe que quand on soumet un échantillon de fer à une série de cycles magnétiques, produits en faisant tourner le champ magnétisant autour du fer à étu lier, ou réciproquement, il faut exercer sur l'arbre de la partie tournante, un isuple constant, indépendant de la vit sse et proportionnel à la perte d'inergie par cycle dans le cas considéré. La mesure de ce couple donne la mesure de l'hystérésis.

L'avteur signale ensuite les analogies et les dissemblances qui existent entre ces deux appareils et permettant d'expliquer les divergences des résultats qu'ils donnent. Il montre que l'hystérésimètre d'Ewing mesure l'hystérésis alternative et que l'appareil de M. Blondel mesure l'hystérésis toumante, celle que l'on trouve dans les induits de dynamos, d'où eatre les deux appareils une différence systématique à peu près constante. Enfin il se résume en disant qu'en fait, la plus grande partie des différences entre ces deux hystérésimètres, abstraction faite de la différence systématique, a pour cıuse l'erreur commise dans le tracé de la graduation.

Influence des régulaleurs sur le fonctionnement en parallile des alternateurs et description d'un noupeau systeme de commande de ces rigulateurs.

Dans le fonctionnement en parallèl des alternateurs, dit M. DE Marchena, le régulateur joue certainement un róle absolument prépondérant. Trop d'importance a d'abord été attachée à la grandeur de l'irrégularité dans un tour et à la valeur del'angle d'écart maxinum. Avec les volants qu'il est d'usage d'adopter, cette irrégularité et cet angle d'écart sont toujours tres faibles et ils ne pourraient donner lieu qu'à des courants de circulation insignifiants. C'est le régulateur oui par une instabilité intempestive ou un manque de proportions entre les divers organes qui le constituent, donne lieu aux impulsions de la force motrice véritablement dangereuses par leur basse fréquence pour la bonne marche en parallele, et cela quei que soit le type de machines et la masse du volant.

Il est de toute importance que la puissance motrice soit régulière pour chaque régime de charge et il faut pour cela que le régulateur agisse avec rapidité, précision, et passe d'une position d'équilibre à l'autre avec le minimum d'oscillations. L'auteur examine alors les lois générales qui régissent le fonctionnement des régulateurs de vitesse en usage; il les classe en deux catégories principales : régulateurs à poids et régulateurs à ressorts et étudie en détail un régulateur type dans chacune de ces catégories; les conclusions auxquelles il arrive ont un caractère général s'appliquant à tous les régulateurs en usage.

M. de Marchena étudie ensuite les conditions à remplir pour que le fonctionnement de ces appareils soit soustrait à tous phénomènes oscillatoires. Et parmi ceux-ci il distingue et analyse successivement:

I. Les oscillations propres du régulateur considéré isolément, c'est-à-dire celles qui se produisent quand le manchon de lappareil arrive à sa position d'équilibre avec une certaine vitesse, ou quand il revient vers cette position après en avoir été écarté d'une certaine quantité. L'analyse mathématique du phénomène montre que l'inertie est, en général, nuisible au bon fonctionnement des régulateurs. Mais cette inertie n'est pas toujours réduite à son minimum parce qu'elle joue, d'autre part, un rôle utile en s'opposant aux trop brusques variations de la vitesse du manchon telles que celles que pourraient produire la réaction des organes de la distribution et l'irrégularité de la vitesse durant un tour. Ainsi, les régulateurs des machines à vapeur doivent posséder une inertie notable. Au contraire, pour les turbines hydrauliques où le régulateur agit par l'intermédiaire de servo-moteurs dont la comnande est très douce et ne donne lieu à aucun choc, et pour lesquelles la puissance motrice s'exerce régulièrement et sans impulsions, l'inertie n'a aucune utilité et lon doit employer, de préférence, des régulateurs ayant leur effort statique produit presque en entier par des ressorts.
11. Les oscillations du şstème constitué par le régulateur et le moteur, celles qui interviennent en cas de variation brusque de la charge, par suite d'un amortissement trop énergique du régulateur. Après avoir passé en revue les conditions à remplir pour que le fonctionnement du régulateur ne solt pas susceptible d'éprouver des oscillations de nature a provoquer des impulsions de la puissance motrice, condition suffisante pour des moteurs actionnant des dynamos a courant continu ou des alternateurs isolés, M. de Marchena fait observer que, pour que le fonctionnement des régulateurs des machines motrices actionnant des alternateurs accouplés en parallele soit tout à tait satisfaisant, Il faut, non seulement qu'sls ne puissent osciller par eux-mèmes, mais encore qu'ils ne soient pas sensibles aux diverses oscillations de la machine qui peuvent prendre naissance indépendamment d'eux, et il étudie l'nfluence que peuvent avoir les mouvements pendulaires d'un moteur sur son régulateur. De cette étude il conclut qu'il y aurait tout intérêt à adopter un régulateur tel que sa púriode d'oscillation soit supérieure à celle de tous les mouvements pendulaires qui peuvent se produire.

Ces mouvements pendulaires sont de deux sortes :

Ceux qui proviennent de l'irrégularité périodique du couple moteur et qui ont, par suite, un caractère permanent;

Ceux qui proviennent des oscillations d s alternateurs provoqués par des variations brusques non périodiques du couple moteur et qui ne durent, par suite, qu'un temps linité après le phénomine qui leur a donné naissance.

En en étudiant les effets, on arrive a voir que, pour amortir les premiers, les machines à vapeur à faible vitesse doivent avoir, de toute nécessité, des régulateurs à grande inertie et des volants lourds; tandis que pour les machines à grande vitesse, on pourra, sans inconvénient, réduire à la fois l'inertie du régulateur et celle du volant.

Pour réduire les oscillations correspondantes du régulateur dans le second des cas précédemment considérés, il faut surtout compter sur l'amortissement. L'auteur indique les divers disposinifs que l'on peut employer à cet effet et en particulier décrit celui qu'il a imaginé et qui consiste à actionner le régulateur non pas directement par la inachine à régler, mais par un petit moteur d'induction branché sur les barres omnibus du tableau. Dans ces conditions, la vitesse du régulateur est entièrement soustraite aux variations píriodiques de vitesse des alternateurs et cet appareil n'entrera en aztion qu'à la suite d'une variation de la charge. Ce système appliqué à une turbine hydraulique de 1000 chevaux a donné d'excellents résultats.

En terminant, $M$. de Marchena fait remarquer que le systeme s'applique aussi bien à l'accouplement en pardlèle d'usines éloignées, hydrauliques ou a vapeur, et qu'il fournit un moyen de réglage simultané de tout l'ensemble des alternateurs accouplés quelles que solent leurs positions relatives. Cette solution a donc un caractère général susceptible de rendre des services dans un grand nombre de cas.

\section{BIBLIOGRAPHIE}

Tout ouvrage dont deux exemplaires seront envoyés à la Rédaction seront analysés dans la Houille Blanche.

L'Electricité et ses Applications, par A. Reboud, licencié ès-sciences mathématiques et physiques; directeur du cours d'Electricité industrielle de Vienne (Isère). Deux volumes in-80 avec de nombreuses figures, - Béranger, Paris.

L'ouvrage que nous présentons à nos lecteurs, écrit sans prétention, débarrassé de tout calcul pénible, réduisant la partie théorique au strict nécessaire, renfermant seulement les principes fondamentaux de la science électrique, mais décrivant soigneusement les applications les plus intéressantes, répond à un véritable besoin. L'essor si rapide de l'electricité explique le nombre prodigieux de publications qui lui sont consacrées : traités purement theoriques s'appuyant 
zur de savantes considérations mathématiques, manuels à l'usage des montcurs et des industriels, livres de pure vulgarisation, périodiques, etc. Et cependant dans cette abondance d'éditions, il est assez difflcile de trouver un ouvrage, d'un prix modéré, qui puisse être utilement placé entre les mains des personnes voulant, sans connaissances spéciales, aborder avec profit les études électrotechniques.

Or, à ce point de vue, l'ouvrage de M. Reboud est hardiment recommandable aux industriels, aux chefs d'ateliers, aux élèves des écoles pratiques de commerce et d'industrie. Il est, à quelques modifications près, le résumé du cours d'électricité industrielle professé, depuis plusieurs années par l'auteur à 'Ecole de Vienne; c'est dire qu'il possède les qualités didactiques des livres destinés à l'enseignement et qui en rendent la lecture facile, précieux avantage que l'on ne saurait trop mettre en évidence lorsqu'il s'agit d'un ouvrage ayant pour unique but de faire pénétrer clairement dans l'esprit des non initiés, les principes fondamentaux de cette scienca qu'll n'est plus permis d'ignorer. Il se recommande, en outre, de la maison d'édition qui nous a habitués à ces livres techniques en lesqueis la perfection de la forme n'a d'égale que les qualités du fond.

L'auteura divisé son travail en deux parties formant chac ane un rolume. Le premier volume, qui a pour titre : Les Piles électriques, senterme d'abord les notions générales de mécanique et de physique, dont la connaissance est nécessaire pour aborder avec fruit l'étude de Télectricité. Les principaux chapitres sont ensuite consacrés à la production de l'électricité statique, l'influence et la condensation; aux machines électro-statiques et à leurs effets; aux piles voltaïques et thermo-électriques; aux effets des courants et à leurs lois; au magnétisme; à l'électro-magnétisme et aux applications des électro-aimants; aux courants d'induction; aux courants de haute fréquence, etc. Enfin, quelques chapitres spéciaux sont consacrés à des applications qui ont eu un retentissement bien justifié ces derniers temps, telles que la télégraphie et la téléphonie sans fils et les rayons $X$.

Le deuxième volume,qui aura pout titre:Les Machines d'induction, traitera de la production, de la distribution et de l'utilisation induszrielle de l'énergie électrique. Nous ne doutons pas qu'il soit aussi bien consu que la première partie et nous souhaitons qu'il ne tarde pas trop à venir la compléter.

\section{L'Oisans. Région de la Meije et du Pelvoux.}

Sous ce titre suggestif, M. Ferrand, l'alpiniste b'en connu, vient do fa re paraitre à la hibrairie Gratier et Rev, un splendide volume in- $4^{\circ}$, de ${ }_{2} 5$ pages, d'un intérêt palpitant puisqu'il raconte toutes les beautés de cette contrée pittoresque entre toutes. Pres de deux cents gravures, imprimées en phototypie, accompagnent le texte et montrent à l'xeil ravi du lecteur les aspects sublimes des hauts sommets, comme la folle course des cascades ou le calme reposant des villiges perdus dans l'Alpe hospitalière et protectrice.

Mieux, peut-ìtre encore, que ses frères ainés : "Les montagnes de la Chartreuse et Bellejonne et Les Sept-Laux ", le nouveau livre de M. Ferrand fait comprendre les grandioses baautés de nos montagnes. L'at délicat et recherché des gravures, leur à-propos pittoresque et leur grâce phototypique, qui résume tous les progrès modernes de la photographie, font de cet ouvrage une ouvre peu banale.

Nous n'avons pas ici à faire l'éloge du style, qui, simple et fort comme les guides de ce pays, escalade sans peine et avee légèreté les cimes les plus hat tes, sans effo:t apparent et avee la delicatesse du pied du chamois.

L'histoire, la physionomie, les anecdotes et les conseils fourmillent et se coudoient, tout en marchant ensemble vers le but, comme une cardyane bien conduite, vers les sommets.

La Meije, le Pelvoux, la Barre des Ecrins, la Grave, le Lautaret et la Bérarde y défilent devant nos yeux comme en un géant cinématographe.

C'est plaisir, pour ceux que leur courage ou leur tempérament a entrainés à travers tous les splendides sites de nos Alpes, de revivre, en lisant ce livre, les heures trop courtes passées au contact intime de la nature.

Et c'est une volupté charmeresse pour les autres, ceux "que leur grandeur retient attachés au rivage», de voyager ainsi, les pieds sous lo table et l'esprit perdu, à travers les hautes régions. Ils pensent vraiment en fermant l'ouvrage y être allés en personne.

N'est-ce pas le meilleur éloge qu'on puisse faire d'un livre sur la montagne.

Nous présentons à M. Henri Ferrand, qui nous a depuis longtemps accoutumés à des ouvres vraiment supéricures, nos plus sincères félicitations pour son nouvel ouvrage l' "Oisans", car, pour une fois, il fait mentir le proverbe : Le mieux est l'ennemi du bien.
Il est peut-être intéressant d'ajouter ici que l'apparition de cet ouvrage constitue une "centieme" peu banale. C'est, en effet, le "centième " des volumes ou brochures que $M$. Ferrand a publiés sur la montagne.

Actuellement, M. Ferrand travaille à son nouveau volume sur le Vercors qui fera suite et terminera cette série d'ouvrages sur le Dauphiné.

Dans cette même collection, paraîtra aussi, l'an prochain, le MontBlanc, par Baud-Bovy.

Tous ces ourrages de grand luxe qui décrivent la montagne sous tous ses aspects, ne manquent pas d intérêt pour les lecteurs de la Houille blanche. lls trouveront là de très utiles documents sur les glaciers, les lacs et les cours d'eau de ces majestueux massifs des Alpes, où réside l'intarissable force, chaque année renouvelée par la nature elle-même.

\section{PUBLICATIONS NOUVELLES FRANĢAISES ET ÉTRANGÈRES}

Endiguement des torrents et amélioration du cours des rivières en hautes montagnes, par E. Dubislav. In-fol. avec plans et figures (Allemand). 55.25

Le Ciment armé et ses applications, par M.-A. Morel, ingénieur. In-1 8 a vec fig. 2.50

Construction du Canal de Jonage. Travaux. Installations hydrauliques et électriques, par R. Chauvin, ingénieur. Grand in- $4^{\circ}$ avec figures et un atlas. 40 francs.

Les Eaux souterraines, par F. Miron. In-8 avec fig., 2.50

Cours d'électricité théorique et pratique professé à l'Ecole nationale d'Arts et Métiers d'Angers, par C. Sarazin, $2^{\circ}$ édition. In-80 avec fig., 20 francs.

Eléments de la science appliquée de l'Electricité, par Tyson Sewell. In 80 (Anglais). 10.50

Ecole de l'Electricité, par G. Schollmeyer. In-80 avec fig. (Allemand), 8.50

Thérie des courants alternatifs et des transformateurs, par J.-L. La Cour. $1^{\text {er }}$ vol. De la iechnique des courants alternatifs. Gr. in-80 avec fig. (Allemand). I 6.50

Manuel pour les installations électriques de haute tension, par Max Jehnke. In-8 avec planches (Allemand), 8.50

Technologie des dynamos, par E. Schulz. In-8 avec fig. (Allemand), 30.50

Recueil de problèmes pour le calcul des conducteurs électriques, par J. Teichmüller, $2^{\circ}$ édit. In-fol. avec fig. (Allemand), 12.50

La Traction électrique, par Paul Dupuy, $2^{\mathrm{e}}$ édition, augmentée d'un appendice. In-80 avec fig., 12 francs.

Trampars électriques et autres en Allemagne, année 1902-03, $6^{\mathrm{e}}$ édition. Gr. in $-8^{\circ}$ (Allemand), 8.50

Recherches sur l'électricité atmosphérique, par Chauveau, 2 vol. in $-4^{\circ}, 12.50$

Introduction à l'étude de l'Electrochimie suivant la théorie électrolytique de la dissociation, par $\mathrm{P}$. Gerdes. Grand in-8 avec fig. (Allemand), 5.50

La préparation du chróme et de sés composés au moren du courant électrique, par Max Le Blanc. $3^{\circ}$ volume des Monographies d'Engelhardt sur l'Electrochinie appliquée. Grand in-80 (Allemand), 8.50

Galvanoplastie et affinage électrique des métaux, par Arnold Philip. In-80 (Anglais), 17.50

Epuration des jus sucrés par l'électricité, par L. Quivy, électrochimiste. In-80, 3.50

L'Imprimeur-Gérant: P. LEgENDRE. 